\title{
Ultracold three-body recombination of fermionic atoms
}

\author{
B.D. Esry ${ }^{1}$, H. Suno ${ }^{1}$, and Chris H. Greene ${ }^{2}$ \\ ${ }^{1}$ Department of Physics, Kansas State University, Manhattan, KS 66506 \\ ${ }^{2}$ Department of Physics and JILA, University of Colorado, Boulder, CO 80309
}

\begin{abstract}
Three-body recombination can play an important role in experiments on ultracold atomic gases because it can produce significant atom loss. Such losses have been seen in Bose-Einstein condensates, but have been thought to play little role for degenerate Fermi gases. We show that under some circumstances, three-body recombination can be just as large in fermi systems as in bose systems. In particular, when there is a two-body resonance near threshold — as is often the case experimentally — the threshold suppression due to the Pauli principle no longer applies.
\end{abstract}

\section{Introduction}

Three-body recombination is the process in which three free atoms collide, producing a diatomic molecule. Schematically, for a generic atom X,

$$
\mathrm{X}+\mathrm{X}+\mathrm{X} \longrightarrow \mathrm{X}_{2}(v, \ell)+\mathrm{X}+E_{v \ell} .
$$

For ultracold collisions, the third atom recedes from the dimer with a relative energy roughly equal to the binding energy of the dimer, $E_{v \ell}$, where $v$ and $\ell$ label the rovibrational state. Just as in any other process that produces a dimer from free atoms, a third body - in this case, another atom - is required in order to conserve energy and momentum.

Three-body recombination is important for experiments on ultracold atomic gases because it can be a leading contributor to atom loss from the trap. The binding energy of the molecule, which gets converted into translational kinetic energy of the molecule and remaining free atom, is typically quite large on the scale of the trap depth. To better understand the role of recombination, one can turn to the rate equation for the density of trapped atoms:

$$
\frac{d n}{d t} \propto-K_{3} n^{3} .
$$

In this expression, $n$ is the density of trapped atoms, and $K_{3}$ is the three-body recombination rate. Of course, the full rate equation for a trapped condensate might also include one-body $(\propto n)$ and two-body $\left(\propto n^{2}\right)$ terms to account for collisions with the background gas or spin-exchange collisions. In the presence of such additional terms, three-body recombination will become a significant effect when either $K_{3}$ or $n$ become large. Since $K_{3} \propto a_{s}^{4}$ ( $a_{s}$ is the two-body $s$-wave scattering length) for three identical bosons $[1,2,3,4], K_{3}$ can become large by making the scattering length large. Experimentally, this adjustment is readily accomplished in both boson and fermion systems by tuning a magnetic field through a Feshbach resonance $[5,6,7,8,9,10]$.

At the extremely low collision energies present in condensates, roughly $100 \mathrm{peV}$, the system is firmly in the quantum mechanical regime. Many quantum mechanical 
effects are thus observable in the recombination cross section - including, for bosons, three-body shape resonances and Stückelberg interferences. Nevertheless, even though three-body recombination has long been studied in the classical regime, few fully quantal treatments exist, and they have only recently begun to appear $[2,3,4]$. To date, however, no theoretical study of recombination of fermions has been undertaken. The present work, then, is the first step in this direction.

\section{Threshold behavior}

In the case of fermions, the Pauli exclusion principle prohibits $s$-wave scattering of atoms in identical spin states, thus leaving only $p$-wave collisions. For two-body collisions, this restriction has the important consequence of suppressing the elastic scattering cross section near threshold, prohibiting the use of evaporative cooling for identical fermions.

Even though recombination of identical fermions is suppressed at ultracold temperatures by the Pauli principle, it does not vanish. In fact, it has been shown that the rate is proportional to $E^{2}$ at low collision energies [11]. The suppression is derived assuming that the system is near threshold. The definition of "near", however, can depend on the low energy two-body scattering parameters. Moreover, the recombination rate can become substantial near a Feshbach resonance. So, in a typical experiment where the system is taken through a resonance at a fixed temperature, the system passes in and out of the threshold regime. The $E^{2}$ threshold law no longer applies near the resonance, and the rate tends to the limit imposed by unitarity - often comparable to or larger than the rates for boson systems. Feshbach resonances are, of course, extremely useful tools for the experimentalist, so understanding the behavior near such a resonance is crucial.

In general, to understand the origin of a scattering threshold law, one needs only to know the long range form of the potential curve. This statement is true for twobody as well as for three-body systems with short range interactions. For two-atom systems, the potentials are obtained from the Born-Oppenheimer approximation, but for three-atom systems, the route to a potential curve is less obvious. We use the adiabatic hyperspherical representation with modified Smith-Whitten hyperspherical coordinates $[3,11,12]$. Simply put, hyperspherical coordinates transform the six relative Cartesian coordinates in the center of mass frame to a set with a single length coordinate, the hyperradius $R$, and five hyperangles. For three identical particles, the hyperradius can be written in terms of the three interparticle distances $r_{i j}$ as

$$
R^{2}=\frac{r_{12}^{2}+r_{23}^{2}+r_{31}^{2}}{\sqrt{3}}
$$

and can thus be thought of as characterizing the overall size of the three-body system. These coordinates also allow us to easily impose the correct permutation symmetry on the wave functions. Solution of the adiabatic equation yields adiabatic hyperspherical potential curves and channel functions. The coupled hyperradial equations are then solved using an $R$-matrix propagation method.

The adiabatic hyperspherical representation reduces the collision of the three atoms to dynamics on a set of coupled hyperradial adiabatic potentials. These potentials bear a strong resemblance to standard molecular Born-Oppenheimer potentials and can be interpreted in much the same way. For instance, Fig. 1 shows the adiabatic 


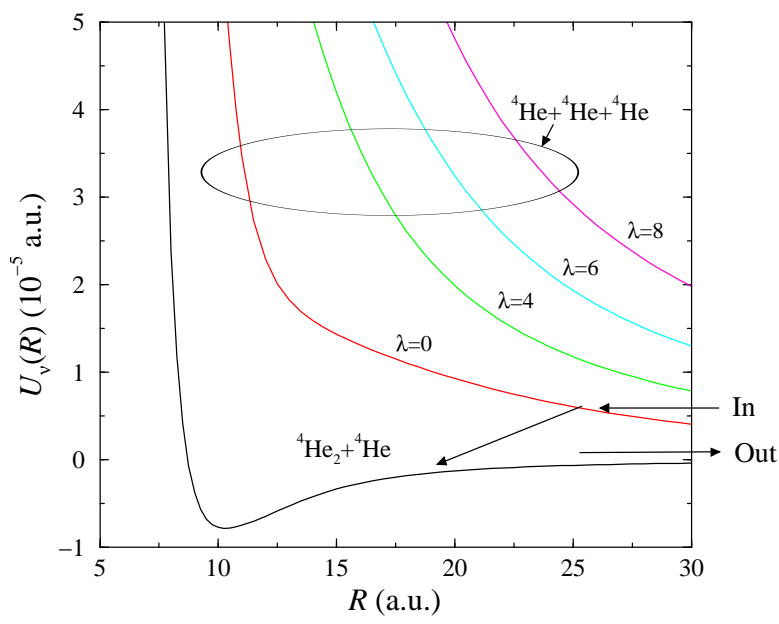

Figure 1: The lowest five adiabatic hypersperical potential curves for three ${ }^{4} \mathrm{He}$ atoms (spin-0 bosons) using a realistic helium-helium interaction (see Ref. [12]). The single recombination channel is labeled as are the three-body continuum channels. The recombination process is schematically indicated with arrows.

hyperspherical potentials for a system of three bosonic ${ }^{4} \mathrm{He}$ atoms. The lowest potential curve in the figure, for instance, correlates to a bound molecule and a free atom far away. For identical fermions, the molecule has unit angular momentum and the corresponding hyperspherical potential has a centrifugal barrier. All of the other curves in Fig. 1 correlate to three free atoms. In fact, there are an infinite number of potential curves associated with three-body continuum channels that approach the three-body breakup threshold $U=0$ asymptotically.

The threshold law is determined by the initial state, which for recombination is the three-body continuum potential. The asymptotic form of these potentials are known to be $[13]$

$$
U(R) \underset{R \rightarrow \infty}{\longrightarrow} \frac{\lambda(\lambda+4)+\frac{15}{4}}{2 \mu R^{2}}
$$

These potentials are thus generalized centrifugal potentials characterized by the quantum number $\lambda$, for which the Wigner threshold analysis is straightforward [11]. Given $\lambda$, the threshold law is

$$
K_{3} \propto E^{\lambda}
$$

as a function of the collision energy $E$. Moreover, it can be shown that upon thermal averaging [14], the threshold law as a function of temperature is

$$
\left\langle K_{3}\right\rangle \propto T^{\lambda} .
$$

Since rates at energies away from threshold are sampled in the thermal averaging, this threshold law will not hold over as large a range in $T$ as Eq. (3) does in $E$. 
Table 1: Summary of threshold laws for all combinations of identical particles. "Identical" here is taken to mean the exact same spin state. (Adapted from Ref. [11].)

\begin{tabular}{lccc}
\hline & $\begin{array}{c}\text { Number of } \\
\text { identical } \\
\text { particles }\end{array}$ & $\begin{array}{c}\text { Dominant } \\
\text { partial wave } \\
\left(J^{\pi}\right)\end{array}$ & Threshold law \\
\hline \hline Bosons & 3 & $0^{+}$ & constant \\
Fermions & 2 & $0^{+}$ & constant \\
& 3 & $1^{+}$ & $E^{2}$ \\
Distinguishable & 2 & $1^{-}$ & $E$ \\
\hline
\end{tabular}

For ultracold collisions, the recombination rate will be dominated by the lowest three-body continuum channel. The value of $\lambda$ associated with this lowest channel depends on the identity of the particles. For instance, the lowest potential for three identical bosons corresponds to $\lambda=0$, giving a constant recombination rate at threshold. For three identical fermions, we find that $\lambda=2$ is the lowest potential, leading to the $E^{2}$ threshold law quoted above. Table 1 summarizes the recombination rate threshold laws for all combinations of identical particles. It is worth noting from the table that the recombination rate is a constant unless identical fermions are present. In the case that there are only two identical fermions - as would be found in a mixture of fermions with different hyperfine states or in a mixture of bosons and fermions - the rate is suppressed by a factor of $E$.

An alternate approach to understanding the threshold law is based on Fermi's Golden Rule [15]. Again, the initial state determines the threshold behavior and can be written in the lab frame as

$$
\Psi_{i}\left(\mathbf{x}_{1}, \mathbf{x}_{2}, \mathbf{x}_{3}\right)=\mathcal{A} e^{i \mathbf{k}_{1} \cdot \mathbf{x}_{1}} e^{i \mathbf{k}_{2} \cdot \mathbf{x}_{2}} e^{i \mathbf{k}_{3} \cdot \mathbf{x}_{3}}
$$

where $\mathcal{A}$ is the antisymmetrization operator. Transforming to center-of-mass and Jacobi coordinates,

$$
\begin{aligned}
\mathbf{X} & =\frac{1}{3}\left(\mathbf{x}_{1}+\mathbf{x}_{2}+\mathbf{x}_{3}\right) \\
\mathbf{x}_{12} & =\mathbf{x}_{2}-\mathbf{x}_{1} \\
\mathbf{x}_{12,3} & =\mathbf{x}_{3}-\frac{1}{2}\left(\mathbf{x}_{1}+\mathbf{x}_{2}\right)
\end{aligned}
$$

gives (up to an overall normalization factor)

$$
\begin{aligned}
\Psi_{i}\left(\mathbf{x}_{1}, \mathbf{x}_{2}, \mathbf{x}_{3}\right)=e^{i \mathbf{K} \cdot \mathbf{x}}\left[e^{i \mathbf{k}_{12,3} \cdot \mathbf{x}_{12,3}} \sin \left(\mathbf{k}_{12} \cdot \mathbf{x}_{12}\right)\right. \\
+e^{i \mathbf{k}_{23,1} \cdot \mathbf{x}_{12,3}} \sin \left(\mathbf{k}_{23} \cdot \mathbf{x}_{12}\right) \\
\left.+e^{i \mathbf{k}_{31,2} \cdot \mathbf{x}_{12,3}} \sin \left(\mathbf{k}_{31} \cdot \mathbf{x}_{12}\right)\right]
\end{aligned}
$$

In this expression, $\mathbf{K}=\mathbf{k}_{1}+\mathbf{k}_{2}+\mathbf{k}_{3}$ is the momentum of the center of mass and can be taken to be zero without any loss of generality; $\mathbf{k}_{i j, k}$ and $\mathbf{k}_{i j}$ are the relative wave 
numbers. Using $\mathbf{K}=0$ and expanding the result for small $\mathbf{k}$ gives

$$
\Psi_{i}\left(\mathbf{x}_{1}, \mathbf{x}_{2}, \mathbf{x}_{3}\right) \underset{k_{i} \rightarrow 0}{\longrightarrow}\left(\mathbf{k}_{1} \cdot \mathbf{x}_{12,3}\right)\left(\mathbf{k}_{2} \cdot \mathbf{x}_{12}\right)-\left(\mathbf{k}_{2} \cdot \mathbf{x}_{12,3}\right)\left(\mathbf{k}_{1} \cdot \mathbf{x}_{12}\right)+\mathcal{O}\left(k^{3}\right) .
$$

Since this result must be squared in Fermi's Golden Rule, its $k^{2}$ dependence translates into an $E^{2}$ dependence of the rate as was found with the adiabatic hyperspherical analysis. One interesting consequence that can be seen from this expression is that the leading $k^{2}$ term vanishes if the vector nature of the quantities is not important. In other words, three-body recombination of identical fermions is suppressed by a factor of $E^{3}$ in one-dimension - and presumably also in quasi-one-dimensional geometries.

\section{Recombination near a two-body resonance}

Given the experimental importance of two-body Feshbach resonances, it is essential to understand three-body recombination under these resonant conditions. For instance, for bosons it is known that $K_{3} \propto a_{s}^{4}$ where $a_{s}$ is the two-body scattering length. While this result does not take into account the mutichannel two-body physics that gives the Feshbach resonance, it can be taken as a first approximation to the physics near the resonance, and this will be our approach below. The recombination rate thus grows rapidly in the vicinity of a Feshbach resonance since $a_{s}$ goes through a pole there. The behavior of the rate for fermions, though, remains a question.

Before we address this question, we must define the low-energy scattering parameter for identical fermions. We will use the two-body $p$-wave "scattering volume", defined as

$$
V_{p}=-\lim _{k \rightarrow 0} \frac{\tan \delta_{p}(k)}{k^{3}}
$$

where $\delta_{p}(k)$ is the $p$-wave scattering phase shift and $k$ is the wave number. The scattering volume $V_{p}$ is related to the $p$-wave scattering length $a_{p}$ (see, for instance, Ref. [16]) by $V_{p}=a_{p}^{3}$. We choose $V_{p}$, rather than $a_{p}$, as the parameter to characterize the three-body recombination of fermions since an artificial nonanalyticity is introduced into $a_{p}$ when taking the cube root of the quantity in the right-hand side of Eq. (7).

When the collision energy is near threshold, the threshold law can be combined with dimensional analysis to yield

$$
K_{3} \propto\left|V_{p}\right|^{\frac{8}{3}}
$$

Since the extent of the threshold regime depends on $V_{p}$, generally becoming more limited with increasing $\left|V_{p}\right|$, this scaling can only be expected to hold at relatively small $\left|V_{p}\right|$. It is worth noting that the recombination rate for bosons is comparatively insensitive to this effect since the rate is a constant at threshold.

Figure 2 shows our results for three-body recombination of identical, spin-polarized fermions. The $\frac{3}{8}$-root of $K_{3}$ is plotted there to reveal the scaling law more clearly. The energy-dependent rate $K_{3}$ (solid line) is indeed linear only for a range of $V_{p}$; in particular, for $V_{p}$ between roughly -4000 and +3500 , with a larger slope on the negative $V_{p}$ side. The thermally-averaged rate (dashed line) obeys the scaling law above for a smaller range of $V_{p}$ as expected. Each symbol in the figure represents 


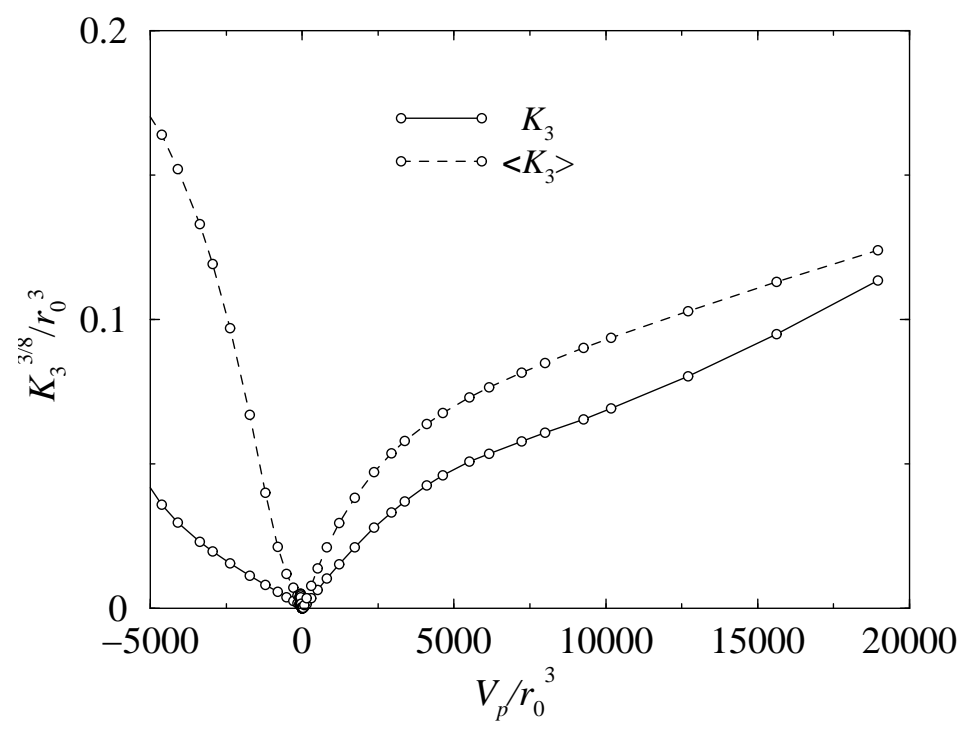

Figure 2: Recombination rate for identical fermions using the model potentials in Eqs. (8) and (9). The parameter $r_{0}$ was taken to be 15 a.u. The solid line shows the energy-dependent rate $K_{3}(E)$ at $E=5 \mu \mathrm{K}$; the dashed line, the thermally averaged rate $\left\langle K_{3}\right\rangle(T)$ at $T=5 \mu \mathrm{K}$.

a separate numerical solution of the three-body scattering problem for the model potential

$$
V=v\left(r_{12}\right)+v\left(r_{23}\right)+v\left(r_{31}\right)
$$

with

$$
v\left(r_{i j}\right)=D \operatorname{sech}^{2}\left(\frac{r_{i j}}{r_{0}}\right)
$$

To obtain the results shown, we fixed $r_{0}$ at 15 a.u. and varied $D$ to change the scattering volume while keeping only a single two-body bound state. We show in Ref. [14] how these results are sufficient to obtain the rates for any value of $r_{0}$, i.e. for any range of the two-body potential.

Several possible features are pointed out in Fig. 2 that are easily understood from the adiabatic hyperspherical picture. It is useful, then, to recall that three-body recombination can be seen as a transition from one three-body continuum channel to a recombination channel, driven by nonadiabatic coupling. Qualitatively, the adiabatic potentials for fermions display analogous behavior, as functions of $V_{p}$, as the adiabatic potentials for bosons show as functions of $a_{s}[17,3]$. Figure 3 shows representative adiabatic hyperspherical potentials and nonadiabatic coupling strengths for both positive and negative $V_{p}$. For both bosons and fermions, the entrance channel goes from being strongly repulsive for positive $V_{p}$ and $a_{s}$ to having an attractive well behind a potential barrier for negative values. Due to permutation symmetry considerations [11], the entrance channel for the fermions is generally more repulsive, however, than the 
addition of a simple $J=1$ centrifugal potential term to the boson curves would imply. The recombination channels are also very similar, although it should be noted that there is no Efimov effect [18] for fermions in the limit $\left|V_{p}\right| \rightarrow \infty$. Efimov physics plays a key role in the interpretation of the ultracold recombination of bosons [3]. Whether there is some other interesting physical effect in this limit remains an open question.

The primary difference between fermion and boson systems lies in the nonadiabatic coupling. While it is still similar for negative $V_{p}$ and $a_{s}$, for positive values the similarities end. Where the coupling strength for bosons shows a definite peak whose position increases linearly in $a_{s}$, the coupling strength for fermions remains peaked at small $R$ with a slowly decaying shoulder whose extent grows in proportion to $V_{p}^{1 / 3}$. For negative values of $V_{p}$, we thus expect that the fermion recombination rate can show enhancement due to resonances localized by the potential barrier (shape resonances) just as for bosons, until the collision energy rises above the top of the barrier - or, as in Fig. 2, the barrier shrinks below the fixed collision energy. In this case, the recombination rate will approach the unitarity limit which is substantial at $5 \mu \mathrm{K}$ with a value of almost $10^{-22} \mathrm{~cm}^{6} / \mathrm{s}$. While we have seen the rapid rise of the rate as the energy approaches the barrier maximum, we have not yet seen evidence of tunneling resonances in our calculations. Since there is a centrifugal barrier in the recombination potential, resonances that can be excited by tunneling through this barrier are

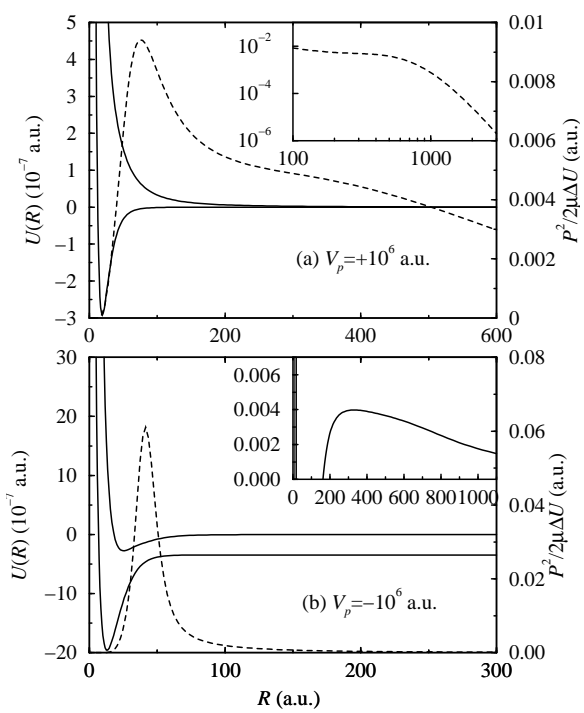

Figure 3: Adiabatic hyperspherical potentials and nonadiabatic coupling strengths for (a) $V_{p}=+10^{6}$ a.u. and (b) $V_{p}=-10^{6}$ a.u. The inset in (a) shows the coupling strength on a log-log scale to emphasize the shoulder that develops. As $V_{p}$ gets larger, this shoulder moves to larger $R$ as $V_{p}^{1 / 3}$. The inset in (b) shows the potential barrier in the entrance channel. Tunneling through this barrier can lead to resonances in the recombination rate. Note that $1 \mathrm{~K}=3.16 \times 10^{-6}$ a.u. also possible - although probably unlikely - for both positive and negative $V_{p}$ when $\left|V_{p}\right|$ is large. In this limit, the top of the barrier in the recombination channel would actually lie above the three-body breakup limit.

\section{Summary}

We have shown that the three-body recombination of fermions need not be negligible in the ultracold limit. The Pauli exclusion principle does lead to a suppression of the 
recombination rate, but this suppression is a purely threshold phenomenon. Should there be a two-body resonance, the threshold law certainly breaks down at energies comparable to the resonance energy if not before. By definition, experimentally useful two-body Feshbach resonances have energies comparable to the collision energy. It can thus be expected that losses due to three-body recombination will generically be large in the vicinity of a Feshbach resonance - even for identical, spin-polarized fermions.

We speculate that there might be exceptions that render recombination ineffective in fermion systems. The first, reducing the effective dimensionality of the system, was already mentioned. We showed that by going to one-dimension, the threshold law was changed from $E^{2}$ to $E^{3}$. It was stated above that the threshold law breaks down near a two-body resonance, but it might still be that the reduced density of final states plays a role near the resonance to partially suppress recombination. The second exception might be recombination in a degenerate Fermi gas. If the binding energy of the molecule is smaller than the Fermi energy, then the kinetic energy of the free atom is not enough to put it above the Fermi sea. Recombination would thus be suppressed by Pauli blocking. A simple estimate valid for $V_{p}>0$ of the value of $V_{p}$ required for Pauli blocking to play a role gives $4 \times 10^{10}$ a.u. which is far off the scale in Fig. 2. In other words, Pauli blocking would likely only play a significant role very near a resonance.

\section{Acknowledgments}

This work was supported in part by the National Science Foundation and the Research Corporation.

\section{References}

[1] P. O. Fedichev et al., Phys. Rev. Lett. 77, 2921 (1996).

[2] E. Nielsen and J.H. Macek, Phys. Rev. Lett. 83, 1566 (1999).

[3] B.D. Esry, C.H. Greene, and J.P. Burke, Jr., Phys. Rev. Lett. 83, 1751 (1999).

[4] P.F. Bedaque, E. Braaten, and H.-W. Hammer, Phys. Rev. Lett. 85, 908 (2000).

[5] T. Loftus et al., Phys. Rev. Lett. 88, 173201 (2002).

[6] K.M. O’Hara et al., Phys. Rev. Lett. 85, 160407 (2000).

[7] For example, S. Inouye et al., Nature (London) 392, 151 (1998).

[8] Ph. Courteille, et al., Phys. Rev. Lett. 81, 69 (1998).

[9] J. L. Roberts, et al., Phys. Rev. Lett.81, 5109 (1998).

[10] S.L. Cornish, et al., Phys. Rev. Lett. 85, 1795 (2000).

[11] B.D. Esry et al., Phys. Rev. A 65 R010705 (2002).

[12] H. Suno et al., Phys. Rev. A 65, 042725 (2002).

[13] E. Nielsen et al., Phys. Rep. 347, 373 (2001).

[14] H. Suno, B.D. Esry, and C.H. Greene, Phys. Rev. Lett. (submitted).

[15] This approach was developed during several discussions with G.V. Shlyapnikov at the conference.

[16] J.L. Bohn, Phys. Rev. A 61, 053409 (2000).

[17] B.D. Esry et al., J. Phys. B 29, L51 (1996).

[18] V.M. Efimov, Phys. Lett. B 33 (1970) 563. 